\title{
Specialist community perinatal screening clinic: service evaluation
}

\author{
Rakesh Magon, ${ }^{1}$ Ruth White ${ }^{2}$
}

The Psychiatrist (2010), 34, 492-495, doi: 10.1192/pb.bp.109.026625

${ }^{1}$ Hertfordshire Partnership Foundation NHS Trust; ${ }^{2}$ Worcestershire Mental Health Partnership NHS Trust

Correspondence to Rahesh Magon (rakesh.magon@hertspartsft.nhs.uk)
Aims and method To identify the outcomes of patients in the obstetric screening clinic. In 2 years, 180 women were referred by midwives to a clinic run by specialist community perinatal team. 'Ultra-high risk' patients were identified. There were four outcome measures predicated on level of care.

Results Of those referred, 69 women were managed in primary care/generic community mental health teams, 90 by specialist perinatal team and 21 did not attend; 23 women were ultra-high risk. The majority of the ultra-high risk patients required treatment with specialist teams.

Clinical implications Specialist community perinatal screening clinics are successful at identifying those at high risk of developing mental health problems. Ultra-high risk women needed a higher level of service. High morbidity in women who fail to attend the services demands more assertive follow-up. Cumulative personal and family history is an important risk factor.

Declaration of interest None.
It is well established that a substantial number of women develop a psychiatric illness in the perinatal period. The development of a psychiatric illness at such a critical time can have adverse effects on the family and the child's social attachments and cognitive development.,

Maternal psychiatric illness is one of the leading causes of maternal deaths., ${ }^{3,4}$ The seventh report of the UK enquiries into maternal deaths, Saving Mothers' Lives, ${ }^{3}$ identified 37 women who died by suicide while pregnant or during their first postnatal year in the UK for the triennium 2003-2005. Compared with the previous two reports, the number of women who died between 2004 and 2005 appeared to have been reduced, but there still seem to be difficulties with the identification and management of psychiatric risk. $^{3}$ The majority of those women who died from suicide or from conditions with a predominately psychiatric aetiology were under psychiatric care, but not from specialists in perinatal psychiatry. ${ }^{3}$ It is suggested in Saving Womens Lives ${ }^{3}$ that generic psychiatric services may not have an adequate awareness of the optimum management of pregnant and postpartum women, and moreover that perhaps if specialist perinatal teams had been involved, some deaths may have been avoided. Such findings strengthen the case for routine perinatal psychosocial screening programmes, with clear referral guidelines and assertive perinatal treatment of significant maternal psychiatric morbidity.

Screening programmes have been introduced in some services, with staff training in identification and management of 'at risk' or symptomatic women. These approaches appear to be successful, but lack clear evidence to validate their usefulness in terms of their psychological outcomes, ${ }^{5}$ consumer satisfaction and cost-effectiveness. ${ }^{6}$ Although sound research is lacking in perinatal psychiatric conditions, $^{7}$ the available research suggests that fairly accurate and feasible screening measures are available. ${ }^{8}$ High prevalence of psychiatric disorders in the perinatal period and availability of effective treatments for these conditions further strengthens the case for screening programmes. ${ }^{9}$ Identification of women 'at risk' (particularly those with bipolar disorder), symptomatic, or with a diagnosis of depression or anxiety, enables early intervention and reduction in psychiatric morbidity in the perinatal period.

With these considerations in mind the specialist community perinatal team (SCPT) in Worcestershire Mental Health Trust has developed an antenatal screening programme. This serves a delivered population of approximately 4500 per year. Initially, midwives were trained by a community nurse with specialist experience in perinatal illness in small group settings. Approximately a year after starting the clinic, the midwives received further training with a particular emphasis on the risk factors for puerperal psychosis and discussion of the then two most recent confidential enquiries into maternal deaths. Senior midwives supported this training but as yet this is not mandatory training. Training for midwives was also disseminated in the midwifery system by the midwifery hierarchy.

The SCPT runs a specialist community perinatal screening clinic in Worcester Royal Hospital for the detection of women who are symptomatic or have 
identifiable risk factors for mental illness. The assessments take place in the antenatal department and involve a structured psychosocial interview including administration of screening tools (Edinburgh Postnatal Depression Scale and Antenatal Risk Questionnaire). ${ }^{10,11}$

Our study aimed to identify the outcomes of patients in the specialist clinic. We compared the outcomes in women attending and those not attending the clinic.

\section{Method}

As a part of a service evaluation of the SCPT, data were collected for all patients who attended and who did not attend the clinic. Respective general practice surgeries were contacted by telephone to study the outcome measures. Outcome measures included:

- no mental health problems

- referred to the community mental health team (CMHT)

- referred to the SCPT

- treatment in primary services with medication and/or counselling.

At the initial booking of an expectant mother, the midwife was asked to identify a woman's personal history of care in secondary psychiatric services or a family history of a first-degree relative with care in secondary services. This served to avoid a midwife having to try to identify particular psychiatric conditions, the latter being performed on assessment by a specialist community psychiatric nurse in a clinic held in the antenatal department at the hospital. Patients invited to the clinic were sent a leaflet with the appointment letter outlining the rationale for the service. The patients assessed in this clinic were later discussed in a multidisciplinary team meeting and the decision was then made as to whether the patient was taken on by the specialist service or discharged to either a CMHT or general practice.

It should be noted that the specialist perinatal service was not available to all women in the study because of the geographical constraints of the service. In these instances patients were referred back to a general practitioner (GP), with recommendations to refer to a general service (in the absence of a perinatal service) or referred directly to a CMHT with recommendations for management.

\section{Results}

In 2 years, 180 women were referred by midwives after initial booking to a specialist community perinatal screening clinic. This identified three groups (Fig. 1): women retained in the SCPT $(n=90)$; women passed back to the primary or generic CMHT care $(n=69)$; and women who did not attend the screening clinic $(n=21)$.

Among those who were referred to primary/generic CMHT care, the majority $(n=41)$ did not develop any mental health problems. However, 19 women did require treatment either in primary $(n=8)$, secondary (CMHT; $n=8)$ or tertiary (SCPT; $n=3$ ) care; 9 outcomes were not studied as the patients had moved out of area.

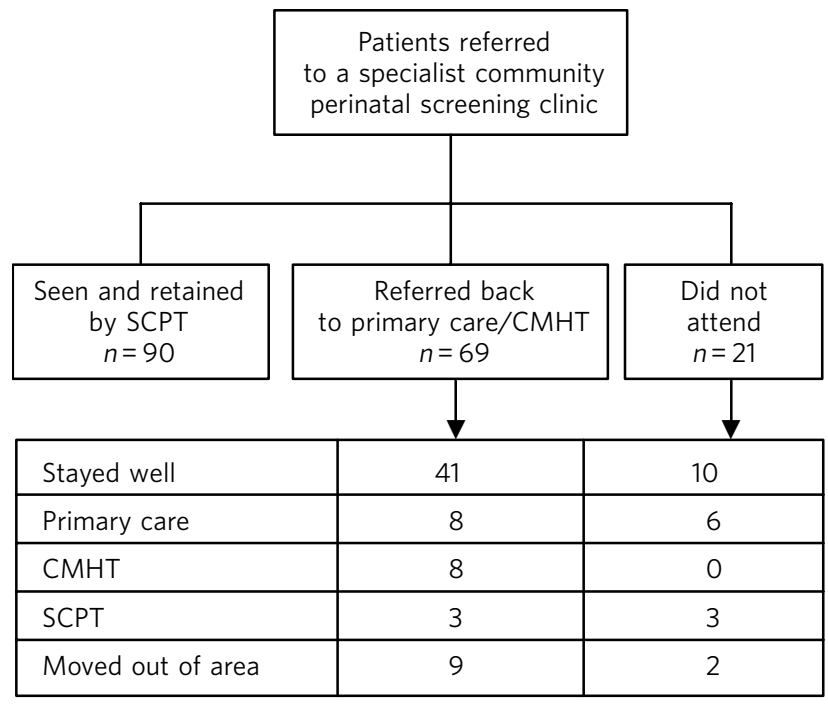

Fig 1 Outcome of patients in the study. CMHT, community mental health team; SCPT, specialist community perinatal team.

Compared with those referred back to primary care or CMHT, nine of those women who did not attend developed mental health problems: six required treatment in primary care and three by the SCPT; two outcomes were not studied as those patients had moved out of area.

Our study also identified a group of women carrying a very high risk of developing mental health problems (Table 1). For the purpose of the study we identified women with a personal history of psychosis and/or a family history of psychosis or suicide as 'ultra-high risk' patients. A total of 23 such women were identified. Of these, 20 attended and 3 did not attend the specialist community perinatal screening clinic. Among those who attended the specialist community perinatal screening clinic, the majority (15/20) were retained within specialist community perinatal services. Of these 20 women, 5 were referred back to primary care $(n=3)$ or to CMHT $(n=2)$.

Of 15 ultra-high risk patients retained in the SCPT, 4 women had a personal history of psychosis, 3 had a family history of psychosis and 8 had both personal and family history of psychosis; 10 women were assertively managed, 3 monitored without intervention and 2 dropped out of treatment.

Of the three ultra-high risk women passed back to primary care, one patient had a personal history of psychosis, one had a family history of psychosis and another one had both personal and family history of psychosis. At their own request, two out of these three women were referred back to primary care. Another woman with a family history of psychosis was referred back to primary care. She was not considered eligible to have services under SCPT as our services did not cover the area. Two out of the three women were later treated by the SCPT.

Two women passed back to CMHT were identified as ultra-high risk patients. One, with both personal and family history of psychosis, was offered SCPT services but chose to remain with her CMHT. However, she later required treatment with the SCPT. Another woman who had a 
Table 1 Outcome of ultra-high risk patients

\begin{tabular}{|c|c|c|c|}
\hline & $\begin{array}{l}\text { Patients with personal } \\
\text { history of psychosis, } n\end{array}$ & $\begin{array}{l}\text { Patients with family history } \\
\text { of psychosis/suicide, } n\end{array}$ & $\begin{array}{c}\text { Patients with personal and family } \\
\text { history of psychosis, } n\end{array}$ \\
\hline $\begin{array}{l}\text { Specialist community perinatal team } \\
\text { (SCPT) }\end{array}$ & $\begin{array}{l}\text { 4: } 2 \text { well with prophylaxis; } \\
1 \text { admitted; } 1 \text { treated at home }\end{array}$ & 3: all remained well & $\begin{array}{l}\text { 8: } 6 \text { required treatment; } \\
2 \text { dropped out of treatment }\end{array}$ \\
\hline General practice & 1: needed treatment in SCPT & 1: remained well & 1: needed treatment in SCPT \\
\hline Community mental health team & 0 & 1: remained well & 1: needed treatment in SCPT \\
\hline Did not attend & 2: both needed treatment in SCPT & 0 & 1: needed treatment in SCPT \\
\hline
\end{tabular}

family history of suicide was referred to CMHT and remained well. She was not considered eligible to have services under SCPT as our services did not cover the area.

Among those who did not attend the specialist clinic $(n=21)$, the initial midwife screening identified three women as ultra-high risk patients (two with personal history of psychosis and one with both personal and family history of psychosis). All three were later treated by SCPT.

\section{Discussion}

In out-patient clinics, between 26 and $50 \%$ of first scheduled appointments result in no shows. ${ }^{12}$ Compared with these findings, the non-attendance rate in our study was low $(11.6 \%, n=21)$. Our data, however, also demonstrate that women who did not attend the clinic had high rates of mental health problems, with almost half of them requiring treatment in primary, secondary or tertiary care. High morbidity in this group demands more assertive follow-up of women who fail to attend initial appointments. Studies conducted in community mental health settings recommend several measures to reduce non-attendance. These include asking patients to make their own appointment, shortening the waiting period for appointments, telephone prompts, orientation and education about treatment. ${ }^{13}$

Our findings are consistent with, and extend, previous research in finding high rates of mental health problems and morbidity in women with personal and/or family history of severe mental illness (the ultra-high risk group in our study). Women who have had a past episode of severe mental illness following delivery have a one-in-two to onein-three chance of recurrence. ${ }^{14}$ In the UK report Saving Mothers' Lives, ${ }^{3} 79$ of the 98 women who died from psychiatric causes had a past psychiatric history and were at risk of recurrence of their disorder, or a relapse of their condition following child birth. The risk factors that consistently show reasonable predictive value, particularly for the development of depression, psychosis and recurrence of bipolar disorder, are past psychiatric history, current disorder, and family history of psychosis. ${ }^{15}$

The majority of ultra-high risk women (15/23) had both personal and family history of psychosis. This finding supports the need to focus on cumulative personal and family history of psychosis and suicide when assessing risks in women in both antenatal and postnatal period. The confidential enquiry into maternal deaths ${ }^{3}$ highlights the need to routinely ask questions about family and personal history of psychiatric disorder at the antenatal clinic. This information enables health professionals to realise the significance of mental health problems arising in the early stages of pregnancy. ${ }^{3}$ Prompt recognition of these risk factors would enable appropriate antenatal interventions in women at risk and prevent psychological morbidity across the perinatal period. The decrease in the rate of deaths from suicide reported in the triennium 2003-2005 may indicate that recommendations made in the previous two reports about identifying women at potential risk in the antenatal period are having a beneficial effect. ${ }^{3}$

Our study highlighted the need for a higher level of service for ultra-high risk women. The majority of women identified as ultra-high risk (20/23) required treatment by the SCPT. The specialist community perinatal service was successful in identifying women at very high risk of developing mental health problems and retained the majority of these women to deliver assertive monitoring and management. For those who were referred back to a GP or CMHT, relevant risks were highlighted and appropriate recommendations were made. In the main, such referral was at the request of the patient.

The study demonstrates that this screening programme is successful in identifying those with a high risk of developing mental health problems perinatally. Identifying at-risk women enables assertive monitoring, early treatment and prevention of admissions to general psychiatric wards or mother and baby units.

Further improvements of early detection and management of perinatal mental illness include providing easy access to services and the careful evaluation of patient satisfaction with screening programmes to encourage participation and involvement of both service users and carers.

\section{About the authors}

Rakesh Magon is Specialty Registrar (ST6) in Psychiatry, Hertfordshire Partnership Foundation NHS Trust, and Ruth White is Consultant Psychiatrist, Worcestershire Mental Health Partnership NHS Trust.

\section{References}

1 Murray L, Stein A. The effects of postnatal depression on the infant. Baillieres Clin Obstet Gynaecol 1989; 3: 921-33.

2 Marmorstein NR, Malone SM, lacono WG. Psychiatric disorders among offspring of depressed mothers: associations with paternal psychopathology. Am J Psychiatry 2004; 161: 1588-94. 
3 Lewis G (ed). Saving Mothers' Lives: Reviewing Maternal Deaths to Make Motherhood Safer, 2003-2005. The Seventh Report on Confidential Enquiries into Maternal Deaths in the United Kingdom (CEMACH). CEMACH, 2007.

4 Sullivan EA, Hall B, King JF. Maternal Deaths in Australia 2003-2005 Australian Institute of Health and Welfare, 2008.

5 Austin MP, Priest SR, Sullivan EA. Antenatal psychosocial assessment for reducing perinatal mental health morbidity. Cochrane Database Syst Rev 2008; 4: CD005124.

6 Buist A, Bilszta J, Milgrom J, Condon J, Speelman C, Hayes B, et al. The Beyondblue National Postnatal Depression Program: Prevention and Early Intervention 2001-2005, Final Report. Beyondblue, 2006.

7 McLennan JD, Offord MD. Should postpartum depression be targeted to improve child mental health? J Am Acad Child Adolesc Psychiatr 2002; 41: 28-35.

8 Gaynes BN, Gavin N, Meltzer-Brody S, Lohr KN, Swinson T, Gartlehner G, et al. Perinatal Depression: Prevalence, Screening Accuracy, and Screening Outcomes. Summary, Evidence Report/Technology Assessment No. 119. Agency for Healthcare Research and Quality, 2005.
9 Buist AQ, Barnett BEW, Milgrom J, Pope S, Condon JT, Ellwood DA. To screen or not to screen - that is the question in perinatal depression. Med J Aust 2002; 177 (suppl 7): s101-5.

10 Cox JL, Holden JM, Sagovsky R. Detection of postnatal depression. Development of the 10-item Edinburgh Postnatal Depression Scale. Br J Psychiatry 1987, 150: 782-6.

11 Austin MP, Priest SR. New developments in perinatal mental health. Acta Psychiatr Scand 2004; 110: 321-2.

12 Allan AT. No-shows at community mental health clinic: a pilot study. Int J Soc Psychiatry 1988; 34: 40-6.

13 Chen A. Noncompliance in community psychiatry: a review of clinical interventions. Hosp Community Psychiatry 1991; 42: 282-6.

14 Oates M. Deaths from psychiatric causes. In Why Mothers Die 20002002: The Sixth Report of the Confidential Enquiries into Maternal Deaths in the United Kingdom (ed G Lewis): 15-73. RCOG Press, 2004.

15 National Collaborating Centre for Mental Health. Antenatal and Postnatal Mental Health: Clinical Management and Service Guidance. National Institute for Health and Clinical Excellence, 2007. 